\title{
High SiO abundance in the HH212 protostellar jet
}

\author{
S. Cabrit ${ }^{1}$, C. Codella ${ }^{2,3}$, F. Gueth ${ }^{4}$, and A. Gusdorf ${ }^{1}$ \\ ${ }^{1}$ LERMA, UMR 8112 du CNRS, Observatoire de Paris, École Normale Supérieure, Université Pierre et Marie Curie, \\ Université de Cergy-Pontoise, 61 Av. de l'Observatoire, 75014 Paris, France \\ e-mail: sylvie.cabrit@obspm.fr \\ 2 INAF, Osservatorio Astrofisico di Arcetri, Largo E. Fermi 5, 50125 Firenze, Italy \\ 3 Institut de Planétologie et d'Astrophysique de Grenoble, UMR 5274, UJF-Grenoble 1/CNRS-INSU, 38041 Grenoble, France \\ ${ }^{4}$ IRAM, 300 rue de la Piscine, 38406 Saint-Martin-d'Hères, France
}

Received 8 June 2012 / Accepted 26 October 2012

\section{ABSTRACT}

\begin{abstract}
Context. Previous $\mathrm{SiO}$ maps of the innermost regions of $\mathrm{HH} 212$ set strong constraints on the structure and origin of this jet. They rule out a fast wide-angle wind, and tentatively favor a magneto-centrifugal disk wind launched out to 0.6 AU.

Aims. We aim to assess the $\mathrm{SiO}$ content at the base of the $\mathrm{HH} 212$ jet to set an independent constraint on the location of the jet launch zone with respect to the dust sublimation radius.

Methods. We present the first sub-arcsecond $\left(0{ }^{\prime} 44 \times 00^{\prime} 96\right)$ CO map of the HH212 jet base, obtained with the IRAM Plateau de Bure Interferometer. Combining this with previous $\mathrm{SiO}(5-4)$ data, we infer the $\mathrm{CO}(2-1)$ opacity and mass-flux in the high-velocity jet and arrive at a much tighter lower limit to the $\mathrm{SiO}$ abundance than possible from the (optically thick) $\mathrm{SiO}$ emission alone.

Results. Gas-phase $\mathrm{SiO}$ at high velocity contains at least $10 \%$ of the elemental silicon if the jet is dusty, and at least $40 \%$ if the jet is dust-free, if $\mathrm{CO}$ and $\mathrm{SiO}$ have similar excitation temperatures. Such a high $\mathrm{SiO}$ content is challenging for current chemical models of both dust-free winds and dusty interstellar shocks.

Conclusions. Updated chemical models (equatorial dust-free winds, highly magnetized dusty shocks) and observations of higher $J$ $\mathrm{CO}$ lines are required to elucidate the dust content and launch radius of the HH212 high-velocity jet.
\end{abstract}

Key words. ISM: individual objects: HH212 - ISM: jets and outflows - ISM: molecules - stars: protostars

\section{Introduction}

The jet origin in accreting young stars and its impact on star and planet formation is still a major enigma. Although a magnetohydrodynamical (MHD) process appears to be required, it is still debated which fraction of the mass-flux originates from the stellar surface, from reconnections in the magnetosphere-disk interaction zone, or from the disk surface, with all three regions probably contributing to some degree (see e.g. Ferreira et al. 2006, for a review). Rotation searches in atomic microjets from $\mathrm{T}$ Tauri stars set an upper limit of $\simeq 0.2-3$ AU on the launching radius of any atomic steady magneto-centrifugal disk wind (Anderson et al. 2003; Ray et al. 2007, and references therein). On the other hand, MHD disk winds launched beyond 0.2-1 AU are expected to be mostly molecular (Panoglou et al. 2012). Therefore the molecular component of jets holds an important clue to the total radial extent of the launch zone.

A key species in this respect is $\mathrm{SiO}$, a specific tracer of molecular jets from the youngest Class 0 protostars, with minimal contamination by ambient swept-up gas (Guilloteau et al. 1992; Gueth et al. 1999; Hirano et al. 2006, 2010). The HH212 jet in Orion (Zinnecker et al. 1998) provides particularly interesting constraints on jet structure. An inner bipolar microjet peaking at $\pm 500-1000 \mathrm{AU}$ from the source was discovered in $\mathrm{SiO}$ (Codella et al. 2007, hereafter Paper I). The fastest SiO gas reaches radial velocities $\simeq \pm 10 \mathrm{~km} \mathrm{~s}^{-1}$ from systemic, i.e. a flow speed $V_{\mathrm{p}} \simeq 150 \mathrm{~km} \mathrm{~s}^{-1}$ after correction for inclination $\left(\simeq 4^{\circ}\right.$ from the plane of the sky; Claussen et al. 1998), arguing that it is tracing material closely associated with the primary jet. This view is supported by its narrow width $\leq 90$ AU (Cabrit et al. 2007, hereafter Paper II) and by the small blue/red overlap in $\mathrm{SiO}$ despite a quasi edge-on view, which restricts the opening angle of the fastest $\mathrm{SiO}$ gas to $<4^{\circ}-6^{\circ}$ (Paper I). At low radial velocities less than $\pm 6 \mathrm{~km} \mathrm{~s}^{-1}$ from systemic, $\mathrm{SiO}$ traces broader structures consistent with expanding jet-driven bowshocks (Lee et al. 2008). The pointed shape of these bowshocks requires that the wind speed drops sharply away from the axis. This appears to be inconsistent with the fast wide-angle wind predicted by the X-wind model (cf. Cai et al. 2008), and requires a slow wideangle wind (if any) more compatible with an extended disk wind.

An extended MHD disk wind also seems favored by the tentative rotation signatures reported across the tip of the $\mathrm{SiO}$ bowshocks. A magneto-centrifugal launch radius of 0.3-0.05 AU was inferred assuming a flow speed $V_{\mathrm{p}}=100-200 \mathrm{~km} \mathrm{~s}^{-1}$ (Lee et al. 2008); but the low mean radial velocity of the rotating gas, $\left|V-V_{\text {sys }}\right|=1.5-4 \mathrm{~km} \mathrm{~s}^{-1}$, implies a lower deprojected flow speed $V_{\mathrm{p}} \simeq 20-60 \mathrm{~km} \mathrm{~s}^{-1}$, hence a larger launch radius of $0.6 \mathrm{AU}$ in both lobes (see Eq. (5) in Anderson et al. 2003), if the rotation interpretation is correct. Unfortunately, no rotation estimates are yet available for the fastest $\mathrm{SiO}$ jet material, as it remains unresolved laterally at 0.'3 resolution (Paper I; Lee et al. 2008).

The $\mathrm{SiO}$ gas-phase abundance may offer an independent clue to the location of the jet origin with respect to the dust sublimation radius $(\simeq 0.2 \mathrm{AU})$, since silicon is one of the main grain constituents. Our previous line ratio analysis in the HH212 microjet shows that $\mathrm{SiO}$ is optically thick for $J_{\text {up }}=2$ to 8 . The implied minimum $\mathrm{SiO}$ abundance strongly depends on the poorly-known 


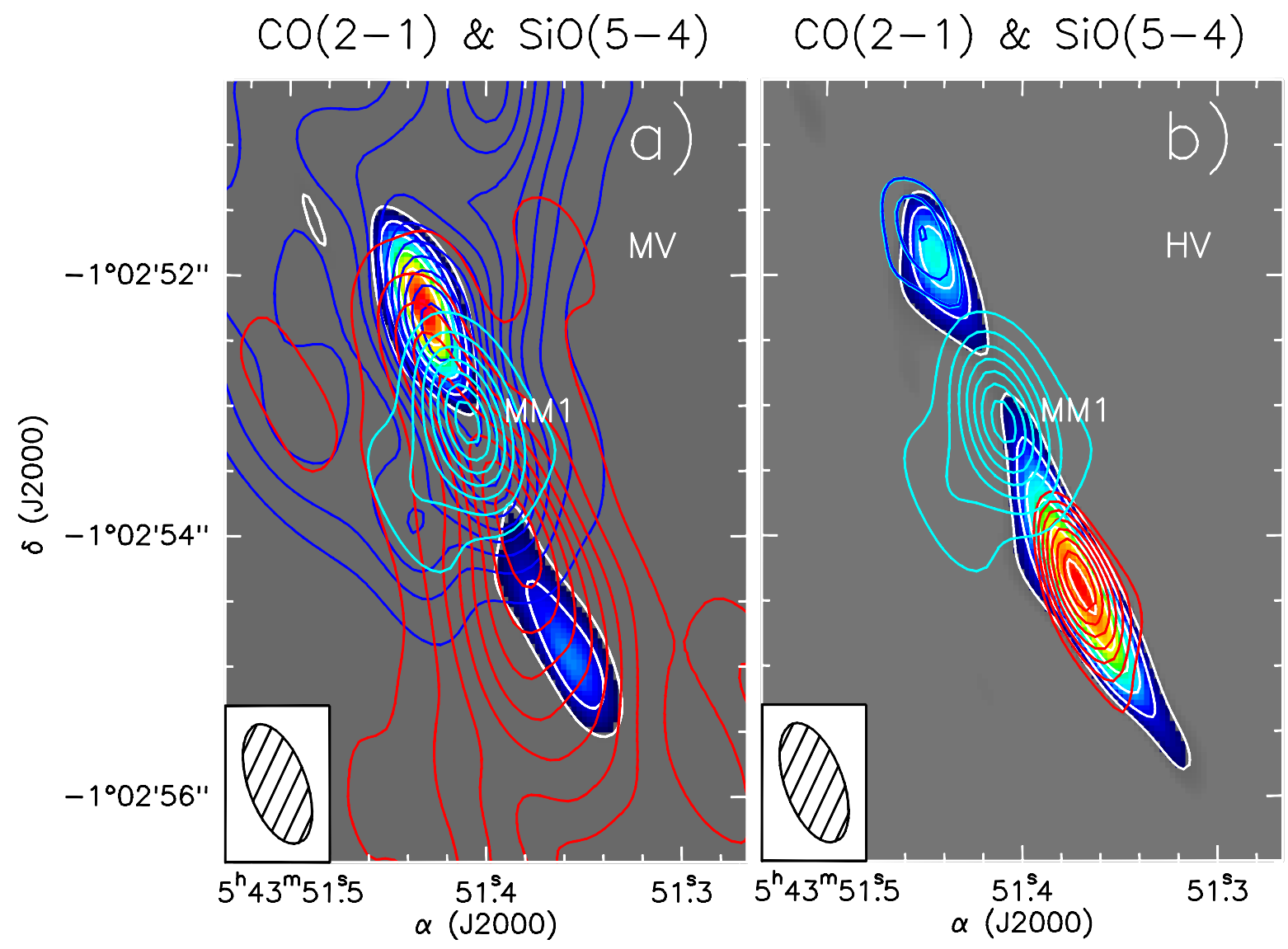

Fig. 1. $\mathrm{CO}(2-1)$ emission (blue and red contours) overlaid on top of $\mathrm{SiO}(5-4)$ emission from Paper I (color scale with white contours). Both maps were cleaned with the same beam of $0^{\prime} 96 \times 00^{\prime} 44$ at $\mathrm{PA}=20^{\circ}$ ( $\mathrm{cf}$. filled ellipse in lower left corner). a) (Left panel) Medium-velocity (MV) emission, integrated over [ $-9.5,-1.5] \mathrm{km} \mathrm{s}^{-1}$ (blue), and $[+3.5,+9.5] \mathrm{km} \mathrm{s}^{-1}$ (red). CO contours range from $6 \sigma\left(13 \mathrm{~K} \mathrm{~km} \mathrm{~s}{ }^{-1}\right)$ to $78 \sigma$ by $6 \sigma$. SiO contours range from $6 \sigma\left(74 \mathrm{~K} \mathrm{~km} \mathrm{~s}^{-1}\right)$ to $18 \sigma$ by $\left.3 \sigma . \mathbf{b}\right)$ (Right panel) High-velocity (HV) emission integrated over [-16.5, -9.5$] \mathrm{km} \mathrm{s}^{-1}$ (blue), and $[+9.5,+12.5] \mathrm{km} \mathrm{s}^{-1}$ (red). CO contours range from $6 \sigma\left(1.4\right.$ and $1.1 \mathrm{~K} \mathrm{~km} \mathrm{~s}^{-1}$ for blue and red, respectively) to $13 \sigma$ by $1 \sigma$. SiO contours range from $6 \sigma\left(21.5 \mathrm{~K} \mathrm{~km} \mathrm{~s}^{-1}\right)$ to $21 \sigma$ by $3 \sigma$. In both panels, $1.4 \mathrm{~mm}$ continuum from the central source MM1 is shown in light-blue contours, ranging from $10 \sigma\left(5 \mathrm{mJy}^{\text {beam }}{ }^{-1}\right)$ to $70 \sigma$ by steps of $10 \sigma$.

jet density and line-of-sight velocity gradient, and varies between $0.05 \%$ and $90 \%$ of the elemental silicon (Paper II). In this letter, we present new $\mathrm{PdBI} \operatorname{CO}(2-1)$ observations of the HH212 microjet, which allow us to put more severe constrains on its density, mass-flux, and $\mathrm{SiO}$ abundance. We compare the latter with chemical models of dust-free vs. dusty winds, and outline the work required to proceed in solving this question.

\section{Observations}

$\mathrm{CO}(2-1)$ observations of the central region of the HH212 outflow were obtained in February 2008 and January, March, and April 2009 with the IRAM Plateau de Bure Interferometer $(\mathrm{PdBI})$ in France. The six-element array was used in its $\mathrm{A}, \mathrm{B}$, and $\mathrm{C}$ configurations (baselines from $48 \mathrm{~m}$ up to $760 \mathrm{~m}$ ) for a total time of $\sim 23 \mathrm{~h}$. The $\mathrm{CO}(2-1)$ line at $230.5380 \mathrm{GHz}$ was observed with a $40 \mathrm{MHz}\left(\sim 50 \mathrm{~km} \mathrm{~s}^{-1}\right)$ bandwidth and a channel sampling of $\sim 0.1 \mathrm{~km} \mathrm{~s}^{-1}$, later smoothed to $1 \mathrm{~km} \mathrm{~s}^{-1}$ to increase the signalto-noise ratio. Two units with a bandwidth of $320 \mathrm{MHz}$ were used to measure the continuum. The data were reduced using the public GILDAS ${ }^{1}$ software. Amplitude and phase were calibrated on observations of $0528+134$ and $0605-085$. The absolute flux density scale was determined on MWC 349 with an estimated uncertainty of $\sim 25 \%$. Line and continuum maps were produced using natural weighting and were restored with a clean beam

1 http://wWw.iram.fr/IRAMFR/GILDAS of $0 .^{\prime} 96 \times 00^{\prime} 44\left(\mathrm{PA}=20^{\circ}\right)$. Since we are interested only in the innermost jet knots within $\pm 2^{\prime \prime}$ of the central source, no correction was applied for primary beam attenuation $\left(\mathrm{HPBW} \simeq 22^{\prime \prime}\right.$ ). The flux, position, and size of the continuum source all agree with the $1.4 \mathrm{~mm}$ data presented in Paper I, except for the tentative source MM2, which is not confirmed. The secondary source seen at $850 \mu \mathrm{m}$ by Lee et al. (2008) at $\Delta \alpha=+1^{\prime \prime}, \Delta \delta=-0.7$ is also unconfirmed (see Fig. 1). In the following, we concentrate on our results in $\mathrm{CO}(2-1)$. Radial velocities are expressed in the $V_{\text {LSR }}$ rest frame unless otherwise specified.

\section{Results}

\subsection{The inner $5^{\prime \prime}$ : outflow cavity vs. jet emission}

Figure 1 compares in two different velocity ranges the cleaned $\mathrm{CO}(2-1)$ map of the inner $5^{\prime \prime}$ of the outflow with the $\mathrm{SiO}(5-4)$ map from Paper I, restored here with the same synthesized beam as for $\mathrm{CO}(2-1)$. In the left-hand panel (Fig. 1a) both lines are summed over a medium-velocity interval (hereafter MV) covering $\left[-9.5 \mathrm{~km} \mathrm{~s}^{-1},-1.5 \mathrm{~km} \mathrm{~s}^{-1}\right]$ in the blue and $\left[+4.5 \mathrm{~km} \mathrm{~s}^{-1}\right.$, $\left.+9.5 \mathrm{~km} \mathrm{~s}^{-1}\right]$ in the red. It can be seen that the CO MV emission peaks along the $\mathrm{SiO}$ axis, but also delineates a broader component not seen in $\mathrm{SiO}$, with a biconical morphology opening away from the exciting source. This corresponds to the base of the CO swept-up cavity mapped on larger scale by Lee et al. (2006, 2007). Owing to this extended component and the lack 


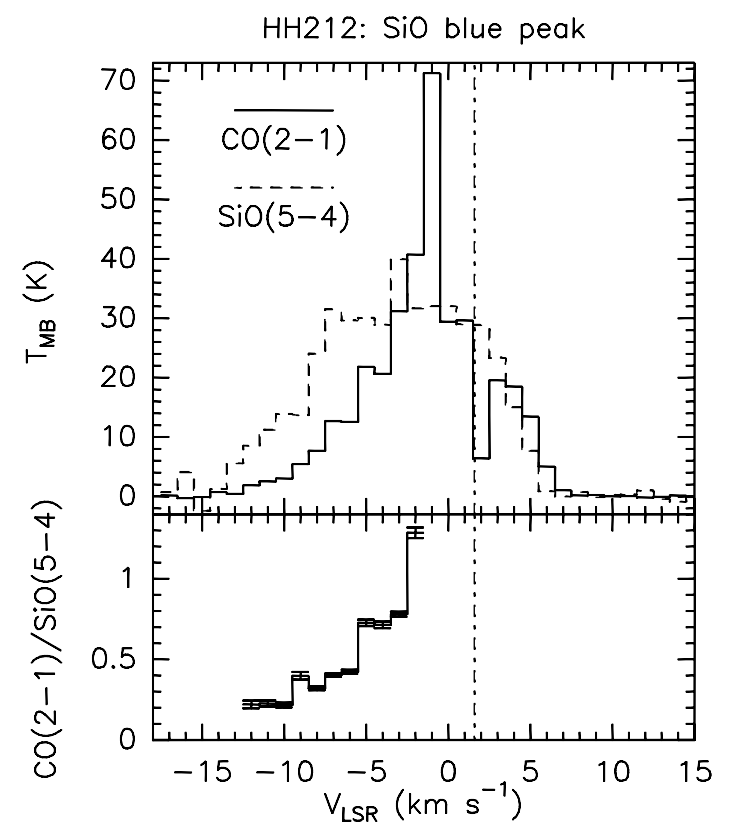

Fig. 2. Top panel: line profiles in $\mathrm{CO}(2-1)$ (solid) and $\mathrm{SiO}(5-4)$ (dashed) toward the northern $\mathrm{SiO}$ peak. Bottom panel: ratio of $\mathrm{CO}(2-$ 1) to $\mathrm{SiO}(5-4)$. The vertical dot-dashed line marks the ambient LSR velocity $\left(+1.6 \mathrm{~km} \mathrm{~s}^{-1}\right.$; Wiseman et al. 2001).

of short spacings in our $u-v$ coverage, the reconstructed $\mathrm{CO}$ brightness in the MV range is very sensitive to the chosen cleaning parameters. In the right-hand panel (Fig. 1b) both lines are summed over a high-velocity interval (hereafter HV) covering $\left[-16.5 \mathrm{~km} \mathrm{~s}^{-1},-9.5 \mathrm{~km} \mathrm{~s}^{-1}\right]$ in the blue and $\left[+9.5 \mathrm{~km} \mathrm{~s}^{-1}\right.$, $+12.5 \mathrm{~km} \mathrm{~s}^{-1}$ ] in the red. In this $\mathrm{HV}$ interval, $\mathrm{CO}$ is seen to trace the same gas as $\mathrm{SiO}$, i.e. a narrow jet still unresolved in the transverse direction, with no significant contamination from the broad cavity. We verified that contrary to the MV emission, the HV emission is compact enough that its flux is robust and independent of cleaning parameters.

\section{2. $\mathrm{CO}(2-1)$ opacity in the HV jet}

As shown in Paper II and confirmed by Lee et al. (2008), $\mathrm{SiO}(5-$ 4 ) is close to LTE and optically thick in the inner HH212 knots. On the other hand, the high ratio $\mathrm{CO}(3-2) / \mathrm{CO}(2-1)>1.5-2$ seen at high velocity (Lee et al. 2007) requires that $\mathrm{CO}(2-1)$ is optically thin in the $\mathrm{HV}$ jet. We may then infer its opacity $\tau_{\mathrm{CO}}^{21}$ from the line temperature ratio $R$ of $\mathrm{CO}(2-1)$ to $\mathrm{SiO}(5-4)$ as $R \simeq \tau_{\mathrm{CO}}^{21}\left(T_{\mathrm{ex}}^{\mathrm{CO}} / T_{\mathrm{ex}}^{\mathrm{SiO}}\right)$, where $T_{\mathrm{ex}}^{\mathrm{CO}}$ and $T_{\mathrm{ex}}^{\mathrm{SiO}}$ are the excitation temperatures of $\mathrm{CO}$ and $\mathrm{SiO}$, respectively. Since $\mathrm{CO}$ has a lower dipole moment than $\mathrm{SiO}$, it is even more easily at LTE and we expect that $T_{\mathrm{ex}}^{\mathrm{CO}} \simeq T_{\mathrm{k}}$. Furthermore, the SiO knots are sufficiently far from the protostar (500 AU) and from any other radiation source that mechanical heating (e.g. shocks, mixing-layers, ambipolar diffusion) will largely dominate over radiative heating (Shang et al. 2002; Panoglou et al. 2012). Therefore, any strong local gradient in $T_{\mathrm{k}}$ should come with a steep accompanying velocity gradient, and we will minimize the difference between $T_{\mathrm{ex}}^{\mathrm{CO}}$ and $T_{\mathrm{ex}}^{\mathrm{SiO}}$ caused by such gradients by examining the ratio $R$ as a function of velocity.

In Fig. 2, we plot the main beam temperature ratio $R$ as a function of velocity toward the (northern) blue $\mathrm{SiO}$ inner knot. In the $\mathrm{HV}$ blue range $V<-9.5 \mathrm{~km} \mathrm{~s}^{-1}$, the $\mathrm{CO}(2-1) / \mathrm{SiO}(5-$ 4) ratio is characterized by a low value $R \simeq 0.2$, and we infer that $\tau_{\mathrm{CO}}^{21}\left(T_{\mathrm{ex}}^{\mathrm{CO}} / T_{\mathrm{ex}}^{\mathrm{SiO}}\right) \simeq 0.2$. In the redshifted $\mathrm{HV}$ range, the ratio $R$ appears similar but $\mathrm{CO}(2-1)$ is affected by a strong dip around $V_{\mathrm{LSR}} \simeq+9 \mathrm{~km} \mathrm{~s}^{-1}$, also seen in $\mathrm{CO}(3-2)$ (Lee et al. 2007). This dip is present at all positions and may be caused by an extended foreground component fully resolved-out by interferometers. Given this complicating circumstance, we focus on the blue HV jet below.

\subsection{HV jet density and mass-flux}

Since the HV blue material is optically thin (Lee et al. 2007), its beam-averaged $\mathrm{CO}$ column density, $N_{\mathrm{CO}}(\mathrm{HVB})$, can be inferred from the $\mathrm{CO}(2-1)$ integrated intensity, without opacity correction. At the northern peak, we measure $T_{\mathrm{mb}} \mathrm{d} V=$ $8.2 \mathrm{~K} \mathrm{~km} \mathrm{~s}^{-1}$ over the $\mathrm{HV}$ range and infer that $N_{\mathrm{CO}}(\mathrm{HVB}) \simeq$ $5 \times 10^{15}\left(T_{\mathrm{ex}}^{\mathrm{CO}} / 50 \mathrm{~K}\right) \mathrm{cm}^{-2}$ for LTE in the range $\simeq 50-500 \mathrm{~K}$ determined in Paper II.

We may also infer a lower limit to the volume density of the HV blue jet at the northern peak. With a jet diameter $D<0$. ' $2=$ $90 \mathrm{AU}$ (cf. Paper II), and a beam size across the jet $b_{\perp}=0 . ' 44=$ $200 \mathrm{AU}$, we find $n_{\mathrm{H}} \simeq\left(b_{\perp} / D^{2}\right) N_{\mathrm{CO}} / X_{\mathrm{CO}}>10^{5}\left(T_{\mathrm{ex}}^{\mathrm{CO}} / 50 \mathrm{~K}\right) \times$ $\left(10^{-4} / X_{\mathrm{CO}}\right) \mathrm{cm}^{-3}$, where $X_{\mathrm{CO}}$ is the $\mathrm{CO}$ gas-phase abundance relative to $\mathrm{H}$ nuclei.

Assuming a steady flow along the jet axis, we may also estimate the one-sided jet mass-flux as given by (see Lee et al. 2007) $\dot{M}_{\mathrm{j}}=1.4 m_{\mathrm{H}} V_{\mathrm{j}} b_{\perp} N_{\mathrm{CO}} X_{\mathrm{CO}}^{-1} \simeq$ $10^{-7}\left(T_{\mathrm{ex}}^{\mathrm{CO}} / 50 \mathrm{~K}\right)\left(10^{-4} / X_{\mathrm{CO}}\right) M_{\odot} \mathrm{yr}^{-1}$, where $V_{\mathrm{j}}=150 \mathrm{~km} \mathrm{~s}^{-1}$ is the relevant deprojected speed for the HV jet (cf. Sect. 1). This $\dot{M}_{\mathrm{j}}$ is ten times lower than the value of Lee et al. (2007) for the same $T_{\mathrm{ex}}^{\mathrm{CO}}$, because we excluded the MV CO emission (dominated by the slower bowshocks and the large-scale cavity). The appropriate choice of $X_{\mathrm{CO}}$ depends on the assumed dust content in the jet. Chemical models of dense Class 0 jets show that $\mathrm{CO}$ self-screens efficiently against FUV photons and contains most of the available gas-phase carbon not locked in grains (Glassgold et al. 1991; Panoglou et al. 2012). Correspondingly, we expect $X_{\mathrm{CO}} \simeq 3.5 \times 10^{-4}$ (solar carbon abundance; Holweger 2001) when the wind is dust-free, and $X_{\mathrm{CO}} \simeq 10^{-4}$ for an interstellar dust/gas ratio $\simeq 1 \%$ (Flower $\&$ Pineau des Forêts 2003). Hence, the above values for $\dot{M}_{\mathrm{j}}$ and $n_{\mathrm{H}}$ are decreased by a factor $\simeq 3.5$ if the HV jet is grain-free, while they both increase by a factor 10 for a high $T_{\mathrm{ex}}^{\mathrm{CO}}=500 \mathrm{~K}$.

\subsection{SiO abundance in the high-velocity jet}

Using the on line RADEX code (Van der Tak et al. 2007) in the large velocity gradient (LVG) approximation, we compared the LTE opacities of $\mathrm{SiO}$ lines versus that of $\mathrm{CO}(2-1)$. For the $\mathrm{SiO}(2-1)$ line and $T_{\mathrm{k}}=50-500 \mathrm{~K}$, we find that

$\frac{X_{\mathrm{SiO}}}{X_{\mathrm{CO}}}=\frac{N(\mathrm{SiO}) / \Delta V}{N(\mathrm{CO}) / \Delta V} \simeq \frac{\tau_{\mathrm{SiO}}^{21} / \tau_{\mathrm{CO}}^{21}}{(122 \pm 10)}\left(\frac{T_{\mathrm{ex}}^{\mathrm{SiO}}}{T_{\mathrm{ex}}^{\mathrm{CO}}}\right)^{1.9}$.

With our result from Sect. 3.2 that $\tau_{\mathrm{CO}}^{21}\left(T_{\mathrm{ex}}^{\mathrm{CO}} / T_{\mathrm{ex}}^{\mathrm{SiO}}\right) \simeq R=0.2$, and the observational constraint $\tau_{\mathrm{SiO}}^{21}>1$ (cf. Paper II), we infer that $X_{\mathrm{SiO}} / X_{\mathrm{CO}}>0.04 \mathrm{f}\left(T_{\mathrm{ex}}^{\mathrm{SiO}} / T_{\mathrm{ex}}^{\mathrm{CO}}\right)^{0.9}$. Adopting the $X_{\mathrm{CO}}$ values discussed above and a solar elemental abundance for silicon $[\mathrm{Si} / \mathrm{H}]_{\odot} \simeq 3.5 \times 10^{-5}$ (Holweger 2001), we obtain that gasphase $\mathrm{SiO}$ in the inner blue jet knot of $\mathrm{HH} 212$ represents at least $\simeq 10 \%$ of the elemental silicon if the wind is dusty, and at least $\simeq 40 \%$ of it if the wind is dust-free, with an uncertainty factor of $\left(T_{\mathrm{ex}}^{\mathrm{SiO}} / T_{\mathrm{ex}}^{\mathrm{CO}}\right)^{0.9}$. The uncertainty in $X_{\mathrm{SiO}}$ from this method is sfactor 2 in planar C-shock models. 


\section{Implications for the launch radius of the $\mathrm{SiO}$ jet}

An $\mathrm{SiO}$ abundance reaching $\geq 10 \%-40 \%$ of elemental silicon is challenging for current models of MHD winds - both dust-free and dusty. We discuss each of these two cases below.

The chemistry of dust-free MHD winds was investigated in $1 \mathrm{D}$ by Glassgold et al. (1991). The wind is launched from a $5000 \mathrm{~K}$ protostar of radius $R_{0}=10 R_{\odot}=0.045 \mathrm{AU}$, and undergoes various degrees of acceleration and expansion. The fraction of silicon in the form of $\mathrm{SiO}$ reaches $\gtrsim 50 \%$ only when the base density exceeds a critical value, corresponding to an isotropic mass-flux rate $\geq 3 \times 10^{-6} M_{\odot} / \mathrm{yr}$ in their accelerating model. This is well above our estimate of $\dot{M}_{\mathrm{j}} \leq 3 \times 10^{-7} M_{\odot} \mathrm{yr}^{-1}$ for the $\mathrm{HV}$ jet in $\mathrm{HH} 212$, if dust free with $T_{\mathrm{k}} \leq 500 \mathrm{~K}$ (see Sect. 3.3). Furthermore, when a flat far-ultraviolet (FUV) excess below $2000 \AA$ is present, $\mathrm{SiO}$ is entirely photodissociated throughout the wind (see Fig. 10 of Glassgold et al. 1991). The assumed FUV photon flux at the $\mathrm{SiO}$ dissociation limit $\simeq 8 \mathrm{eV}$ is similar to that produced by an accretion shock of blackbody temperature $10000 \mathrm{~K}$ and total luminosity $3.5 \mathrm{~L}_{\odot}$. The Class 0 protostar of $14 L_{\odot}$ driving the $\mathrm{HH} 212$ outflow (Claussen et al. 1998) should have a FUV flux from accretion at least as high, hence negligible $\mathrm{SiO}$ in the wind. Increasing the launch radius would not solve the problem, since both the base wind density and the radiation field would drop as $1 / R_{0}^{2}$, keeping the same ratio of $\mathrm{SiO}$ reformation to photodissociation rates. Therefore, spherical dust-free winds do not seem able to reproduce the high $\mathrm{SiO}$ content in the $\mathrm{HH} 212$ jet.

A possible factor favoring $\mathrm{SiO}$ synthesis in dust-free winds would be if the jet does not arise from $4 \pi R_{0}^{2}$ but from a narrow equatorial annulus of width $\Delta R \ll R_{0}$, e.g. as assumed in the $\mathrm{X}$-wind model (Cai et al. 2008). This would increase the base density by a large factor $R_{0} / \Delta R$ for the same jet mass-flux, thus enhancing $\mathrm{Si}^{+}$recombination and $\mathrm{SiO}$ formation with respect to photodissociation. However, this hollow wind geometry would also enable the penetration of destructive FUV photons through the emptied polar regions (see Panoglou et al. 2012), an effect not included in the 1D calculations of Glassgold et al. (1991). Detailed chemical wind models in 2D with a FUV accretion excess are thus necessary to check whether an equatorial dust-free wind could reach an $\mathrm{SiO}$ abundance $\gtrsim 40 \%$ of elemental $\mathrm{Si}$, as estimated in the HV jet of HH 212.

An alternative scenario is that $\mathrm{SiO}$ would form by shock processing of silicate grains in a dusty wind launched from the disk (Chandler et al. 2001; Panoglou et al. 2012). Recent models of C-type shocks with ion-neutral decoupling suggest that sputtering of charged grains by drifting neutrals can release up to $5 \%$ of $\mathrm{Si}$ in the gas phase, which then reacts with $\mathrm{O}_{2}$ or $\mathrm{OH}$ to form $\mathrm{SiO}$ (Schilke et al. 1995; Gusdorf et al. 2008a). A possible problem with sputtering, raised in Paper II, is that the dynamical timescale for the inner $\mathrm{SiO}$ knots in $\mathrm{HH} 212$ is only $25 \mathrm{yr}$, which may be too short to complete the sputtering process and the conversion of $\mathrm{Si}$ into $\mathrm{SiO}$ (Gusdorf et al. 2008b). Fortunately, at the high densities $\geq 10^{5} \mathrm{~cm}^{-3}$ of the HH212 inner knots (cf. Sect. 3.3), grain-grain collisions also become important (Caselli et al. 1997). They will alleviate this problem in two ways: first, by shattering dust grains into smaller fragments, they increase the coupling between charged and neutral fluids and shorten the C-shock timescale. Second, they induce grain vaporization, which directly releases $\mathrm{SiO}$ molecules into the gas-phase, reaching up to $5 \%$ of the total silicon at $40 \mathrm{~km} \mathrm{~s}^{-1}$ (see Guillet et al. 2011). The same process may also occur in single-fluid (J-type) shocks, with $\mathrm{SiO}$ reaching $2 \%$ of the total silicon at $50 \mathrm{~km} \mathrm{~s}^{-1}$ (Guillet et al. 2009). We note that the above
$\mathrm{SiO}$ yields refer only to interstellar shock models with Alfvén speeds $V_{\mathrm{A}}$ of a few $\mathrm{km} \mathrm{s}^{-1}$, a value $\simeq 10$ times lower than expected at $500 \mathrm{AU}$ in protostellar jets (Hartigan et al. 2007; Garcia et al. 2001). Extrapolating the trends with $V_{\mathrm{A}}$ shown in Fig. 5 of Caselli et al. (1997) and Fig. 6 of Guillet et al. (2009) suggests that in dusty MHD jets, grain-grain collisions might be able to release $\gtrsim 10 \%$ of $\mathrm{Si}$ in the form of $\mathrm{SiO}$, compatible with our estimate in HH212 in the dusty wind case. Dedicated shock models with appropriate initial conditions would be necessary to verify this extrapolation.

In conclusion, $\gtrsim 10 \%-40 \%$ of elemental silicon in the form of $\mathrm{SiO}$ is challenging for current chemical models of both dustfree winds and dusty shocks, and calls for additional modeling with more realistic assumptions to clarify the maximum $\mathrm{SiO}$ abundance that can be reached in either case. ALMA observations in $\mathrm{CO}(3-2)$ and $\mathrm{CO}(6-5)$ will also be essential for better constraining the jet temperature, width, mass-flux, and density, as well as the ratio $T_{\mathrm{ex}}^{\mathrm{SiO}} / T_{\mathrm{ex}}^{\mathrm{CO}}$ entering our $\mathrm{SiO}$ abundance estimate, all of which are key parameters for distinguishing among current MHD jet models.

Acknowledgements. We are grateful to G. Pineau des Forêts and M. Tafalla for useful discussions, to an anonymous referee for comments that helped to strengthen the paper, and to the IRAM staff for their support with observations. This research has made use of NASA's Astrophysics Data System, and received financial support from the Programme National de Physico-Chimie du Milieu Interstellaire (PCMI). The IRAM Plateau de Bure Interferometer is funded by INSU/CNRS (France), MPG (Germany) and IGN (Spain).

\section{References}

Anderson, J. M., Li, Z.-Y., Krasnopolsky, R., \& Blandford, R. D. 2003, ApJ, 590, L107

Cabrit, S., Codella, C., Gueth, F., et al. 2007, A\&A, 468, L29 (Paper II)

Cai, M. J., Shang, H., Lin, H.-H., \& Shu, F. H. 2008, ApJ, 672, 489

Caselli, P., Hartquist, T. W., \& Havnes, O. 1997, A\&A, 322, 296

Chandler, C., \& Richer, J. S. 2001, ApJ, 555, 139

Claussen, M. J., Marvel, K. B., Wootten, A., \& Wilking, B. A. 1998, ApJ, 507, L79

Codella, C., Cabrit, S., Gueth, F., et al. 2007, A\&A, 462, L53 (Paper I)

Ferreira, J., Dougados, C., \& Cabrit, S. 2006, A\&A, 453, 785

Flower, D., \& Pineau des Forêts, G. 2003, MNRAS, 343, 390

Garcia, P. J. V., Ferreira, J., Cabrit, S., \& Binette, L. 2001, A\&A, 377, 589

Glassgold, A., Mamon, G. A., \& Huggins, P. J. 1991, ApJ, 373, 254

Gueth, F., \& Guilloteau, S. 1999, A\&A, 343, 571

Guillet, V., Jones, A. P., \& Pineau des Forêts, G. 2009, A\&A, 497, 145

Guillet, V., Pineau des Forêts, G., \& Jones, A. P. 2011, A\&A, 527, A123

Guilloteau, S., Bachiller, R., Fuente, A., \& Lucas, R. 1992, A\&A, 265, L49

Gusdorf, A., Cabrit, S., Flower, D. R., \& Pineau des Forêts, G. 2008a, A\&A, 482, 809

Gusdorf, A., Pineau des Forêts, G., Cabrit, S., \& Flower, D. R. 2008b, A\&A, 490, 695

Hartigan, P., Frank, A., Varnière, P., \& Blackman, E. G. 2007, ApJ, 661, 910

Hirano, N., Liu, S.-Y., Shang, H., et al. 2006, ApJ, 636, L141

Hirano, N., Ho, P. P. T., Liu, S.-Y., et al. 2010, ApJ, 717, 58

Holweger, H. 2001, AIP Conf. Proc., 598, 23

Lee, C.-F., Ho, P., Beuther, H., et al. 2006, ApJ, 639, 292

Lee, C.-F., Ho, P. T. P, Hirano, N., et al. 2007, ApJ, 659, 499

Lee, C.-F., Ho, P. T. P., Bourke, T. L., et al. 2008, ApJ, 685, 1026

McCaughrean, M., Zinnecker, H., Andersen, M., Meeus, G., \& Lodieu, N. 2002, The Messenger, 109, 28

Panoglou, D., Cabrit, S., Pineau des Forêts, G., et al. 2012, A\&A, 538, A2

Ray, T., Dougados, C., Bacciotti, F., Eisloeffel, J., \& Chrysostomou, A. 2007, in Protostars and Planets V, eds. B. Reipurth, D. Jewitt, \& K. Keil, 231

Schilke, P., Walmsley, C. M., Pineau des Forêts, G., \& Flower, D. R. 1997, A\&A, 321,293

Shang, H., Glassgold, A. E., \& Shu, F. H. 2002, ApJ, 564, 853

Van der Tak, F. F. S., Black, J. H., Schier, F. L., Jansen, D. J., \& van Dishoeck, E. F. 2007, A\&A, 468, 627

Wiseman, J., Wootten, A., Zinnecker, H., \& McCaughrean, M. 2001, ApJ, 550, L87

Zinnecker, H., McCaughrean, M. J., \& Rayner, J. T. 1998, Nature, 394, 862 